\title{
Structural Health Monitoring System based on Wireless Sensor Network
}

\author{
Zhifen Yuan \\ Department of Computer and Science, Yunyang Teachers' College, Shiyan 442000, China. \\ 464547@qq.com
}

Keywords: Structural health monitoring (SHM), Wireless sensor networks (WSNs), NS2, Routing protocol.

\begin{abstract}
Firstly, current development of SHM technology and application of WSNs technology in SHM are introduced. Secondly, based on analysis and comparison with routing protocols for WSNs, considering large numbers of nodes, individual nodes with extremely limited resource and data remotely transmission in a WSNs for large structural health monitoring. A highly efficient energy-saving routing protocol named WSPSHM (Wireless Sensor Protocol for Structural Health Monitoring) is developed, and details of routing algorithm are presented. Finally, the network simulation software NS2 is briefly introduced. Test and performance evaluation of routing algorithm with NS2 are discussed. Detailed flow and analyzing results of simulation are presented. Compared with current routing protocols, WSPSHM could effectively reduced radio frequency and ensure that the entire networks have higher throughput and longer life cycle at low level of energy consumption. Feasibility of network organizing technology for WSNs of large structural health monitoring is demonstrated.
\end{abstract}

\section{Introduction}

In recent years, with the rapid development of wireless sensor networks (WSNs), it has become a bridge which connects the physical world with the computer world. [1] Wireless sensor networks not only have the advantages like low wiring cost, high monitoring precision, good fault-tolerance, etc., it also can be monitored remotely. It has outstanding merits in fault diagnosis, security monitoring, and so on. Since the building security accidents happened frequently, how to take real-time and effective monitoring for building's health status, avoid the building security accidents caused by significant structure or equipment damage, fire or building indoor environment change, and provide timely and scientific guidance to health and maintenance of a building have get more and more people's attention. Hence, it is of great theoretical significance and social benefits to set a building security monitoring system based on wireless sensor networks.

As structural health monitoring (SHM) technology for lame civil structures has become a worldwide hot topic, design and instrumentation of structural health monitoring system for existing structures is received more and more attention by the researchers. Smart structural health monitoring (SSHM) technology based on wireless sensor networks (WSNs) providing a new method for structural status information acquisition, safe operation of structure and system instrumentation. Considering network organization technology for large-scale wireless sensor network of lame structural health monitoring, a highly efficient enemy-saving wireless sensor networks routing protocol is investigated and developed. [2]

Combination of wireless sensor network (WSN) and structural health monitoring (SHM)has attracted increasing attention. Relevant research shows that, in most of the WSN applications the major energy intensive operation is transmitting a large amount of data for a long distance. Compare to transmission cost the computation cost can be ignored, so previous research focused on reducing transmission energy. [3] In SHM, it tends to create far more amount of data than other WSN applications. It is necessary to process raw data in-network, through reduce the amount of data need to be trans而tied, energy can be saved. However, in-network processing needs uniform analysis on sample data from mint 1e nodes. The significant large amount of data, complexity of the algorithm 
and the Limited computing power of the wireless sensor nodes make the computation energy become a problem worth to pay close attention to.

\section{Wireless Sensor Networks Technology and Theory Analysis}

This paper analyzes the current situations, progress and challenges faced by the building security monitoring research, summarizes the system structure, characteristics and technical requirements of wireless sensor networks, researches the design and implementation of wireless sensor networks in the fields of building structure health monitoring, building fire security monitoring and building indoor environmental monitoring. System structure OS structure of wireless sensor networks are shown in Fig. 1.

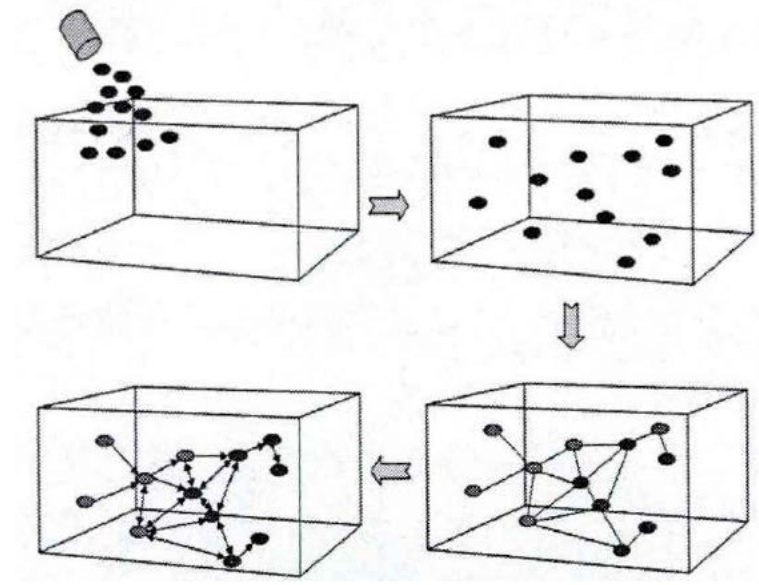

Fig. 1 System structure OS structure of wireless sensor networks

The traditional building indoor environmental monitoring system generates lots of data to transmit. [4] However, there is only limited spectrum resource and power energy. To deal with this problem, this dissertation builds a spectrum sensing mode and proposes a spectrum sensing algorithm based on supporting vector machine. The proposed scheme can sense the available spectrum resource accurately. With the sensed spectrum resource, the throughput and transmission efficiency improve remarkably. Meanwhile, this dissertation also proposes an improved DV-hop algorithm based on compressive sensing theory. The proposed algorithm could reduce the number of samples, save power energy consumption, and improve network lifetime, while without any performance degradation of the networks. (Eq. (1))

$$
\sum_{i=1}^{m} \lambda_{i} / S u m \geq R
$$

For structural global monitoring, the electronics components are compared, analysis and selected. On this base, the design motorization method is used for designing and debugging sensing disposal module, micro-processing module, wireless transceiver module and power module, and thus a wireless acceleration sensor node with digital interface is integrated using the above modules. The interface and a emendations of wireless acceleration sensor are researched importantly. The designed wireless acceleration sensor has such many characteristics as initiation, low-energy consumed, low cost, high reliability, and the democrat experiment shows that it can meet the acceleration measurement requirements in civil engineering. (Eq. (2))

$$
Y_{k}=\sum_{i=1}^{p} b_{i}{ }^{D B} Y_{k-i}+r_{k}^{D B}
$$

Considering that the volume of traditional acquisition equipment is big and that the lead with high-precision is needed, the project of wireless strain collection and transmission system applied for local monitoring is presented, and the system is integrated. Especially, the amplifying disposal module of wireless strain sensor is taken into account importantly. The designed wireless strain collection and transmission system has such characteristic as miniaturization. It can overcome the lacks of the traditional wired system. (Eq. (3)) 


$$
D=\sum_{i=1}^{p}\left(b_{i}^{D B}-b_{i}\right)^{2}
$$

\section{Routing and Power Management in Structural Health Monitoring System}

Due to the large number of time and resource consumption required by the traditional building structural health monitoring system, this dissertation tries to develop a lightweight monitoring system based on WSNs. A genetic ant colony algorithm is proposed to realize multicast routing and multi-hop routing, so as to improve the quality of service and stability of the networks. An improved ant colony algorithm is utilized to save the energy consumption of the networks, so as to enlarge the network lifetime. Node-to-node delivery ratio $g$ different values are shown in Fig. 2.

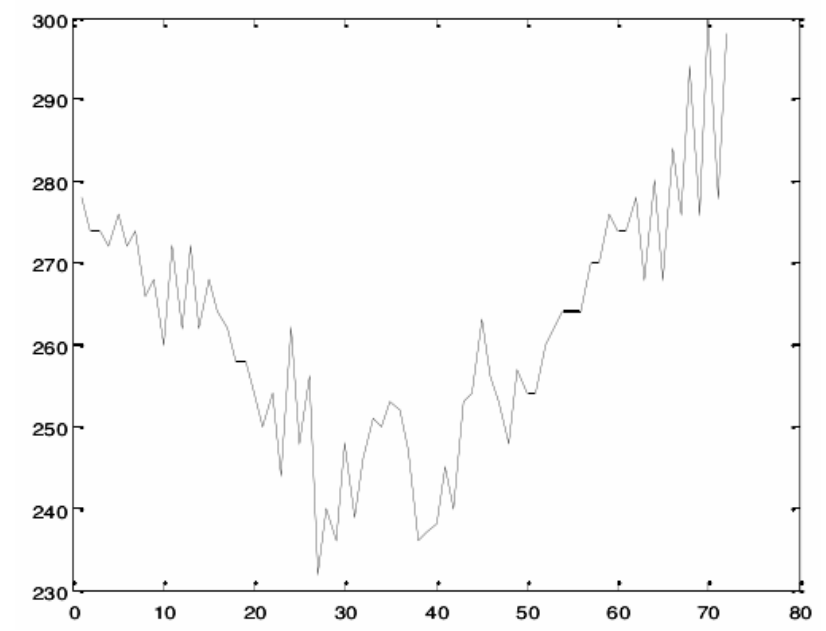

Fig. 2 Different values $(g)$

Table 1 Experiment parameters

\begin{tabular}{cc}
\hline parameters & value \\
\hline Network grid & $(0,0)-(450,450)$ \\
Node number & 200 \\
Eelec & 50 \\
Eda & 5 \\
Message size & 4000 \\
Initial energy distribution & {$[1,7]$} \\
Round number & $-[1,2500]$ \\
\hline
\end{tabular}

Many experimental research of wireless sensor network for SHM is finished. the strain change of reinforced concrete beams in regular static load is also inspected by wireless strain sensor system, and the data collected is disposed of in fusion technique, the experimental result shows that wireless strain sensor system can measure the local status of reinforced concrete beams and reflect the relation of static strain and loads reliably; the pendulum movement of large scale heavy derrick lay barge's hook model installed reducing-pendulum-movement control system is monitored wirelessly, the analysis indicates that the pendulum movement of large scale heavy derrick lay barge's hook model can be monitored rightly using wireless sensing technique and that the given reducing-pendulum-movement control system can lighten the pendulum movement of hook model is validated; the wireless testing on an offshore platform is finished while it endures from ice, and the global change of Shenzhen Dewing Plaza are also monitored using wireless sensor network, the experimental results display that wireless acceleration sensors and their network can monitor rightly the global status of offshore platforms and super high-building. Experiment parameters are shown in Table 1. The calculation results are shown in Fig. 3. 

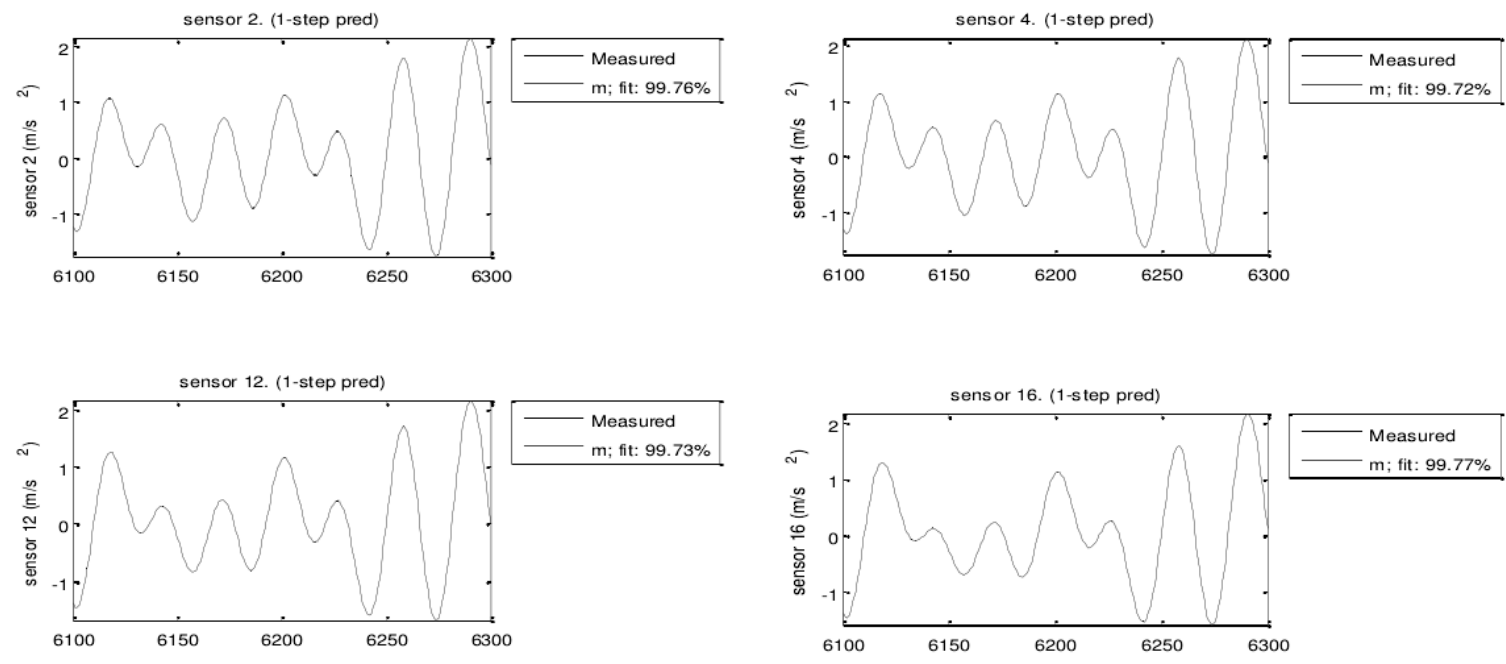

Fig. 3 The calculation results

To deal with the problem of limited function, complex architecture, and high bandwidth requirement in current building fire security monitoring system, this dissertation proposes a novel fire and flammable gas detection WSNs system based on multiple agents theory. A dynamic scheduling scheme is proposed to solve the large scale computing problem involved in the multi-tasks scheduling, so as to reduce the running time and improve the efficiency of task scheduling. A novel D-S data fusing algorithm is utilized to deal with the real time data set, so as to improve the accuracy and stability of the monitoring alarm system, and decrease the false positive and non-response rate as well.

\section{Conclusions}

In this paper, wireless sensors and their network system are developed for the purpose of SHM on the basis of sensing, information disposal and wireless communication technique. The smart sensor Micaz was researched. The work includes compiling program for acceleration collection and being compared with wired sensors in the experiment of testing vibration character of cantilever beam. The results showing that the wireless network consisted by many smart sensors can finish data collection. By analyzing the signals, the frequency of the structure can be identified. To verify the feasibility of the distributed system, ten Imote2s were installed on a concrete-filled steel tube arch bridge, three sensor communities was constructed to measure acceleration. The result shows the effective of the data collection system, each level node can perform successfully. In conclusion, the realization of distributed data acquisition system paved the way for SHM on Imote2s. Distributed computing strategy is considered suitable for implemented on network of densely deployed smart sensor.

\section{References}

[1] Constantinos Marios Angelopoulos,Sotiris Nikoletseas, Theofanis P. Raptis .Wireless energy transfer in sensor networks with adaptive, limited knowledge protocols. Computer Networks, 2014, 70(9): 113-141.

[2] Z.D. Chen, C. Qiao, Y.H. Qiu et al. Dynamics stability in wireless sensor networks active defense mode l. Journal of Computer and System Sciences, 2414, 80(8): 1534-1548.

[3] Ozel O, Tutuncuoglu K, Yang J, et al. Transmission with energy harvesting nodes in fading wireless channels: Optimal policies se-lected areas in communication. IEEE Journal on Selected Areas in Communications, 2011, 29(8): 1732-1743.

[4] Baron Gorain, Partha Sarathi Mandal. Approximation algorithms for sweep coverage in wireless sensor networks. Journal of Parallel and Distributed Computing, 2014, 74(8): 2699-2707. 